Draft Version October 29, 2018

Preprint typeset using IATEX style emulateapj v. 5/2/11

\title{
ALFALFA DISCOVERY OF THE NEARBY GAS-RICH DWARF GALAXY LEO P. I. HI OBSERVATIONS
}

\author{
Riccardo Giovanelli ${ }^{1}$, Martha P. Haynes ${ }^{1}$, Elizabeth A.K. Adams ${ }^{1}$, John M. Cannon ${ }^{2}$, Katherine L. Rhode ${ }^{3}$, \\ John J. Salzer ${ }^{3}$, Evan D. Skillman ${ }^{4}$, Elijah Z. Bernstein-Cooper ${ }^{2}$ and Kristen B. W. McQuinn ${ }^{4}$ \\ Draft version October 29, 2018
}

\begin{abstract}
The discovery of a previously unknown $21 \mathrm{~cm}$ HI line source identified as an ultra-compact high velocity cloud in the ALFALFA survey is reported. The HI detection is barely resolved by the Arecibo $305 \mathrm{~m}$ telescope $\sim 4^{\prime}$ beam and has a narrow HI linewidth (HPFW of $24 \mathrm{~km} \mathrm{~s}^{-1}$ ). Further HI observations at Arecibo and with the VLA corroborate the ALFALFA HI detection, provide an estimate of the HI radius, $\sim 1^{\prime}$ at the $5 \times 10^{19} \mathrm{~cm}^{-2}$ isophote, and show the cloud to exhibit rotation with an amplitude of $\simeq 9.0 \pm 1.5 \mathrm{~km} \mathrm{~s}^{-1}$. In other papers, Rhode et al. (2013) show the HI source to have a resolved stellar counterpart and ongoing star forming activity, while Skillman et al. (2013) reveal it as having extremely low metallicity: $12+\log (O / H)=7.16 \pm 0.04$. The HI mass to stellar mass ratio of the object is found to be 2.6. We use the Tully-Fisher template relation in its baryonic form (McGaugh 2012) to obtain a distance estimate $D_{M p c}=1.3_{-0.5}^{+0.9}$. Additional constraints on the distance are also provided by the optical data of Rhode et al. (2013) and McQuinn et al. (private communication), both indicating a distance in the range of 1.5 to $2.0 \mathrm{Mpc}$. The three estimates are compatible within their errors. The object appears to be located beyond the dynamical boundaries of, but still in close proximity to the Local Group. Its pristine properties are consistent with the sedate environment of its location. At a nominal distance of $1.75 \mathrm{Mpc}$, it would have an HI mass of $\simeq 1.0 \times 10^{6} M_{\odot}$, a stellar mass of $\simeq 3.6 \times 10^{5} M_{\odot}$, and a dynamical mass within the HI radius of $\simeq 1.5 \times 10^{7} M_{\odot}$. This discovery supports the idea that optically faint — or altogether dark - low mass halos may be detectable through their non-stellar baryons.
\end{abstract}

Subject headings: galaxies: spiral — galaxies: distances and redshifts — galaxies: halos — galaxies: luminosity function, mass function — galaxies: photometry — radio lines: galaxies

\section{INTRODUCTION}

The faint end of the luminosity function of galaxies is generally modeled by a power law which is significantly shallower than that prescribed by the $\Lambda \mathrm{CDM}$ scenario for the low end of the mass function of dark matter halos. When this mismatch between observations and theory is found in the vicinity of massive galaxies such as the Milky Way (MW), it is referred to as the "missing satellite problem" (Klypin et al. 1999); when encountered in regions of very low galaxy density, it is referred to as the "void phenomenon" (Peebles 2001). Here, we shall refer to the issue generically as the "dwarf galaxy problem": the observed underabundance of low-mass galaxies relative to theoretical expectations. The conflict between observations and theory is also encountered in the behavior of the HI mass function (Martin et al.|2010), the rotational velocity width function (Papastergis et al. 2011), and the baryonic mass function of galaxies (Papastergis et al. 2012). The numerous ultra-faint dwarf galaxies found

\footnotetext{
${ }^{1}$ Center for Radiophysics and Space Research, Space Sciences Building, Cornell University, Ithaca, NY 14853. e-mail: riccardo@astro.cornell.edu, haynes@astro.cornell.edu, betsey@astro.cornell.edu

2 Department of Physics and Astronomy, Macalester College, Saint Paul, MN 55105. e-mail: jcannon@macalester.edu, ebernste@macalester.edu

3 Department of Astronomy, Indiana University, Bloomington, IN 47405. e-mail: rhode@astro.indiana.edu, slaz@astro.indiana.edu

${ }^{4}$ Minnesota Institute for Astrophysics, University of Minnesota, Minneapolis, MN 55455. e-mail: skillman@astro.umn.edu, kmcquinn@astro.umn.edu
}

in the vicinity of the MW and M31 discovered over the last decade in wide-field optical surveys have alleviated somewhat - but by no means resolved - the mismatch between theory and observations (Koposov et al. 2008). Moreover, the ultra-faint dwarf satellites of the MW are located at small galactocentric distances, well inside the virial radius of the MW, and thus their structural and evolutionary properties have been drastically affected by tidal and hydrodynamical processes (Sand et al. 2012), making it difficult to assess their primitive characteristics.

The possibility that HI observations might help resolve the dwarf galaxy problem was proposed in two influential papers more than a decade ago (Blitz et al. 1999; Braun \& Burton 1999), in which the HI high velocity clouds (HVCs) were invoked as baryonic but starless counterparts to low-mass dark matter halos in the Local Group. In particular, the more compact of those HVCs (sizes $\lesssim 2^{\circ}$ ) were proposed as representative of the relatively primordial, yet undisturbed population of LG low-mass halos. This interpretation was challenged by Sternberg et al. (2002, hereafter SMW02), on the grounds that, if placed at typical LG distances $(\sim 1$ $\mathrm{Mpc}$ ): (i) the sizes of even the more compact HVCs (CHVCs) known at the time were too large (sizes > $10 \mathrm{kpc}$ ) to match the mass-concentration relation of $\Lambda$ CDM halos; and (ii) the CHVCs would be too massive $\left(M_{H I}>10^{7} M_{\odot}\right)$ to explain the lack of detection of similar populations in nearby groups of galaxies (Pisano et al. 2007). The existence of gas-bearing but 
starless (or nearly so) minihalos was recently revived by Giovanelli et al. (2010, hereafter G+10) who identified a category of even more compact HVCs (sizes $\lesssim 10^{\prime}$, thus the ultra-compact HVCs: UCHVCs) in the ALFALFA extragalactic HI survey (Giovanelli et al. 2005). The thermal models of SMW02 are given for a broad range of intergalactic medium (IGM) pressures. While we don't know the specific environmental conditions at the location of any single cloud, plausible models can be fit to the UCHVC data. Placed to distances of a few $\mathrm{Mpc}$, their HI masses would be smaller than detection limits of extant surveys of nearby groups. The one critical item preventing the validation of the idea that the UCHVCs can be counterparts of extragalactic minihalos is that their distances are not known. There are two main routes to advancing such validation; one would be the detection of stellar populations associated with the UCHVCs; the other would be the detection of a population of objects with comparable HI properties in a nearby group of galaxies, clearly associated by virtue of similarity in the distribution of radial velocities. Here we report on a finding that allows for progress along the former route. The object involved is a dwarf galaxy located in the immediate vicinity of the LG.

\section{DISCOVERY OF HI102145.0+180501}

Making use of the seven-horn Arecibo L-band Feed Array (ALFA), the Arecibo Legacy Fast ALFA Survey ALFALFA (Giovanelli et al. 2005) is a blind survey in the HI $21 \mathrm{~cm}$ line covering $7000 \mathrm{deg}^{2}$ of high Galactic latitude sky in two regions: a "Spring" northern Galactic circumpolar region between $7.5 h$ and $16.5 h$ in R.A. and a "Fall" southern Galactic region between $22 h$ and $03 h$ in R.A. Both footprints extend from $0^{\circ}$ to $36^{\circ}$ in Declination. The spectral coverage extends from -2000 to $+18000 \mathrm{~km} \mathrm{~s}^{-1}$ with a channel separation of $\sim 5.5$ $\mathrm{km} \mathrm{s}^{-1}$. Initiated in 2005 and completed in 2012, survey observations were carried out with the $305 \mathrm{~m}$ telescope at the Arecibo Observatory 5 with an angular resolution of $\sim 4^{\prime}$ and an integration time of $\sim 40$ sec per beam, yielding an r.m.s. flux sensitivity of $\sim 2.5 \mathrm{mJy}$ for a spectral feature smoothed to $11 \mathrm{~km} \mathrm{~s}^{-1}$. A catalog containing sources over nearly $3000 \mathrm{deg}^{2} \quad$ (40\% of the total ALFALFA sky) is in the public domain (Haynes et al. 2011).

As discussed in previous works (Haynes et al. 2011 and references therein), the ALFALFA survey observations consist of a series of drift scans which are combined to construct 3-D data cubes. Data processing is carried out sequentially on stripes $2^{\circ} .4$ wide in Declination. Signal identification is performed by applying a matched filtering algorithm in the Fourier domain (Saintonge 2007). The process of catalog construction also includes the inspection of optical databases and the assignment of the most probable optical counterpart to each HI signal.

During the course of construction of the ALFALFA catalog along the Spring stripe centered at Dec. $=+17^{\circ}$, an HI source with heliocentric velocity of $264 \mathrm{~km} \mathrm{~s}^{-1}$ was detected at 102145.0+180501 (epoch 2000.0). The source

\footnotetext{
5 The Arecibo Observatory is operated by SRI International under a cooperative agreement with the National Science Foundation (AST-1100968), and in alliance with Ana G. Méndez-Universidad Metropolitana and the Universities Space Research Association.
}

was designated as an UCHVC of the type reported by $\mathrm{G}+10$. The HI emission appeared to be very weakly, if at all, resolved by the $4^{\prime}$ ALFA beam. Although no cataloged optical galaxy is recorded near the HI position, a very faint blue object appears in both the SDSS and DSS2 blue images, $\sim 20^{\prime \prime}$ NE of the ALFALFA centroid, but within the positional error box. The SDSS image exhibits an irregular, lumpy light distribution that could be described as being marginally resolved into stars. In fact, what we now identify as a single galaxy is seen as a combination of several photometric objects in the SDSS database, some of which are classified as stars and some as galaxies. It is identified as a compact group of galaxies (SDSSCGB11269) by McConnachie et al. (2009) who used an automated search algorithm to identify such objects in the SDSS DR6 catalog. As those authors note, spurious identification of compact groups can be due to errors in the photometric catalog. In fact, this group candidate is included in their less reliable "catalog B". Aided by the good position match with that of the HI source, however, the visual inspection of both the SDSS and the DSS2 blue images suggested an alternative interpretation: that of a very faint, nearby, star forming dwarf galaxy. Follow-up optical observations reported by Rhode et al. (2013): (i) confirmed that the HI source has a stellar counterpart, (ii) provided an accurate optical flux, partly resolved into individual stars, (iii) detected $\mathrm{H} \alpha$ emission indicative of current star formation activity and (iv) produced an independent estimate of the galaxy's distance. Later optical spectroscopy of the H II region revealed the galaxy's extremely low metallicity (Skillman et al. 2013). We shall hereafter refer to it as Leo $\mathrm{P}$ because of its apparently pristine nature. It is also identified as AGC208583 in the catalog maintained by $\mathrm{MPH}$ and $\mathrm{RG}$ and reported in a number of on-line extragalactic data bases.

\section{PROPERTIES OF THE GALAXY LEO P}

The follow-up observations mentioned above include BVR broad-band imaging obtained in excellent seeing conditions $\left(0.6^{\prime \prime}-0.8^{\prime \prime}\right)$ with the WIYN 3.5-m telescope and more recently with the Large Binocular telescope (LBT); $\mathrm{H} \alpha$ imaging with the $2.1 \mathrm{~m} \mathrm{KPNO}$ telescope; optical spectroscopy with the $4 \mathrm{~m}$ KPNO telescope and the Large Binocular Telescope (LBT); and HI synthesis imaging with the Karl G. Jansky Very Large Array ( $V L A 6)$. Ongoing fitting of thermal structure models will yield insights into the phases of Leo P's interstellar medium (Faerman et al., in preparation). Throughout this paper, properties of Leo $\mathrm{P}$ will be tabulated through their explicit dependence on the object's distance $D_{M p c}$ in Mpc. As we discuss in Sections 3.1 and 3.3, that distance is likely to be between 1.5 and $2 \mathrm{Mpc}$.

\subsection{Summary of Optical Properties}

Using the WIYN telescope broad-band imaging data, Rhode et al. (2013) clearly detected the stellar population associated with Leo $\mathrm{P}$, showed that it extends to a radius of $\simeq 225 D_{M p c}$ pc and has a stellar mass of $1.2 \times 10^{5} D_{M p c}^{2} M_{\odot}$. They also obtained constraints to

6 The National Radio Astronomy Observatory is a facility of the National Science Foundation operated under cooperative agreement by Associated Universities, Inc. 


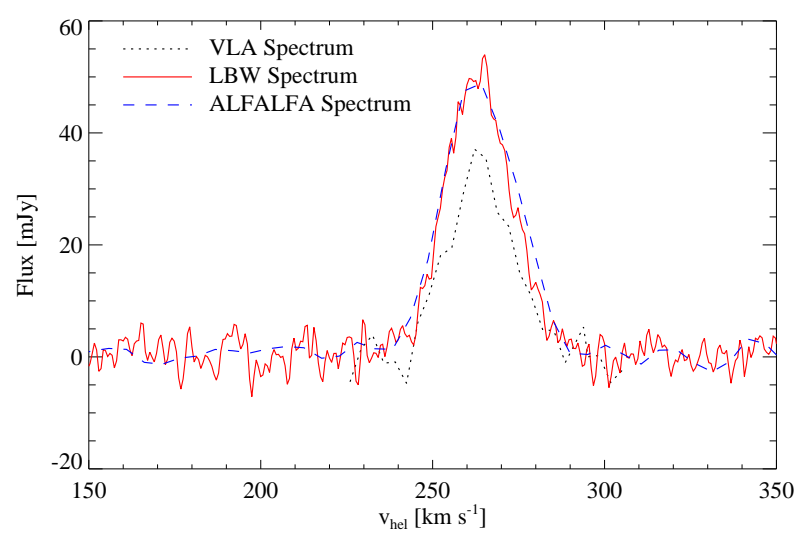

Figure 1. HI line spectra of Leo $\mathrm{P}$ from separate observations: the original ALFALFA spectrum (dashed blue line), the higher resolution spectrum from the targeted LBW observation (solid red line), and the spatially integrated spectrum extracted from the masked VLA cube used to create the moment map in Fig. 2 (dotted black line). The LBW observation matches the ALFALFA spectrum very closely and, despite its substantially higher spectral resolution, it does not reveal any additional spectral detail. The VLA integrated spectrum, sampled at $3.3 \mathrm{~km} \mathrm{~s}^{-1}$, shows missing flux relative to the single-dish observations.

the distance $D_{M p c}$ of Leo P. After extraction of a sample of $\simeq 10^{2}$ resolved stars, a color-magnitude diagram allowed an identification of the red giant stellar branch (RGB), to which isochrones could be fitted. However, the sparseness of the stellar population, coupled with the limited depth of their photometric data, makes the determination of the location of the branch tip (TRGB) difficult. Recently, deeper optical broad band images of the Leo P field have been obtained with the LBT by K. McQuinn et al. (private communication). These data allow a clear identification of the RGB but, again, due to the sparseness of the resolved stellar population, the location of the TRGB cannot yet be sharply defined. Both groups do however agree that the analysis of their respective data sets indicate that the distance to Leo $\mathrm{P}$ lies somewhere between 1.5 and $2.0 \mathrm{Mpc}$. These estimates are complemented by, and in agreement with that of $D_{M p c}=1.3_{-0.5}^{+0.9}$ obtained using the baryonic TullyFisher relation, presented in Section 3.3. Optical spectroscopy data of Skillman et al. (2013) obtained with the LBT shows Leo $\mathrm{P}$ to have the extremely low metallicity of $12+\log (\mathrm{O} / \mathrm{H})=7.16 \pm 0.04$, and to exhibit essentially primordial Helium abundance.

\subsection{HI Properties}

Figure 1 displays the HI spectral profile of Leo $\mathrm{P}$ as extracted from the ALFALFA dataset, traced by the dashed blue line. In March 2012, a single-pixel spectrum was obtained using the Arecibo telescope and the ' $\mathrm{L}$ band-wide' (LBW) receiver with a velocity resolution of $1.2 \mathrm{~km} \mathrm{~s}^{-1}$ (after Hanning smoothing); the on-source integration time was 3 minutes. This spectrum is displayed as the solid red line in Figure 1] it matches and corroborates the ALFALFA spectrum. Since a single pointing recovers practically all the flux of the ALFALFA map, the source angular diameter must be significantly smaller than the $\mathrm{FWHM}=4^{\prime}$ Arecibo beam; however, close inspection of the ALFALFA map suggests that the Arecibo

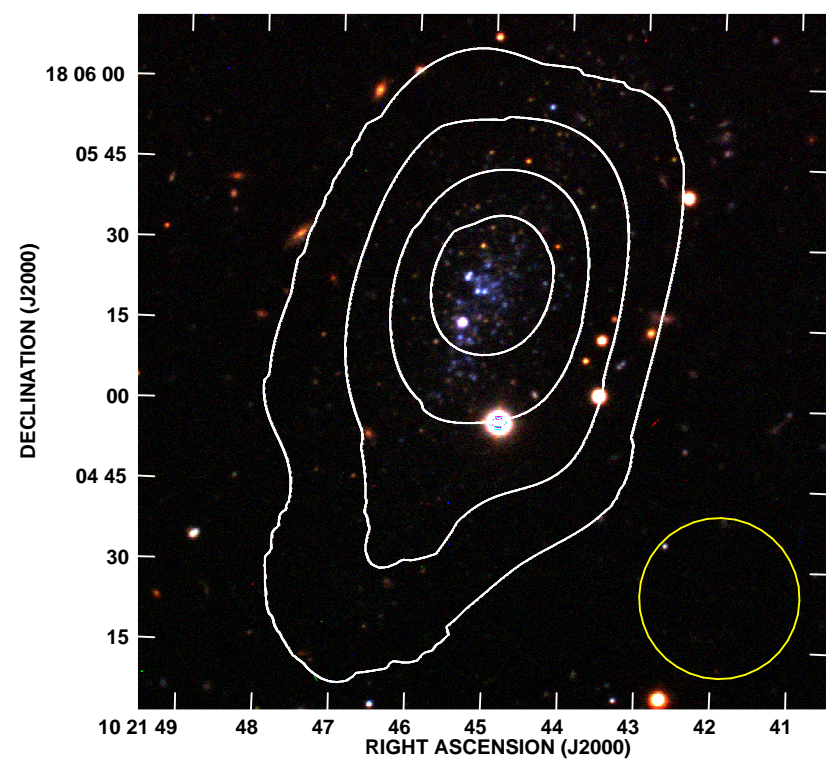

Figure 2. VLA-C integrated HI synthesis preliminary image of Leo $\mathrm{P}$, smoothed to an angular resolution of $30^{\prime \prime}$ (shown as the yellow circle in the lower right), superimposed on the WIYN optical image of Rhode et al. (2013). HI column density contours are shown at $0.5 \times 10^{20} \mathrm{~cm}^{-2}$ intervals, starting at $0.5 \times 10^{20} \mathrm{~cm}^{-2}$ for the outermost contour.

beam starts to resolve a weak extension of the source to the SE. It is unlikely to be due to telescope beam sidelobes. Thus, a rough estimate of the half-mass HI radius between $0^{\prime} .8$ and $1^{\prime} .5$ can be made. No indication of a narrow component of width less than $10 \mathrm{~km} \mathrm{~s}^{-1}$ is evident in either line profile.

In April 2012, 5 hours of VLA observations in the C configuration were obtained as part of the Observatory Director's Discretionary Time. A resulting HI image, smoothed to an angular resolution of $30^{\prime \prime}$, is shown in the form of contours of HI column density overlaid on the WIYN 3.5m optical image in Figure 2] The peak column density is $\sim 2.3 \times 10^{20} \mathrm{~cm}^{-2}$ and the HI major axis radius is $1^{\prime}$ at the column density level of $5 \times 10^{19}$ $\mathrm{cm}^{-2}$. However, only half of the ALFALFA flux is recovered in the VLA image (including flux rescaling: see Jorsater \& van Moorsel 1995) at 30" resolution, indicating that much of the HI detected by ALFALFA is in a diffuse component more extended than that scale.

Figure 1 displays the VLA spectrum integrated over the whole source (dotted line), which is vaguely suggestive of having two spectral components: one as broad as indicated by the Arecibo observations, and a second, narrower than $10 \mathrm{~km} \mathrm{~s}^{-1}$ FWHM. If confirmed, such a bimodality could be attributed to the presence of two thermally stable phases of the atomic gas: a cold $(\mathrm{T}<1000$ $\mathrm{K})$ neutral medium (CNM) associated with the spectrally narrow component that could arise from a single dense, star-forming region, and a warm ( $\mathrm{T}>6000 \mathrm{~K})$, predominantly neutral medium (WNM), enveloping the former, associated with the spectrally broad component (Young \& Lo 1996, Warren et al. 2012). The relative masses of the two components could be used to constrain thermal models of the ISM. The extant observations cannot reliably provide such a constraint yet. They do however provide evidence for a rotating disk. 
Smoothed to an angular resolution of $30^{\prime \prime}$ and a spectral resolution of $3.3 \mathrm{~km} \mathrm{~s}^{-1}$, the VLA-C data reveal a sustained velocity gradient along the major axis (with position angle of $34^{\circ}$ clockwise from North) of the column density distribution shown in Figure 2. While the signal-to-noise is poor, a rotation curve can be extracted from the image. It is shown in Figure 3. No ordered velocity gradient is evident along the minor axis. The rotational velocity amplitude $V_{\text {rot }}$ that can be measured from Figure 3, uncorrected for orbital plane inclination, is $9.0 \pm 1.5 \mathrm{~km} \mathrm{~s}^{-1}$. Assuming that disk inclination can be inferred from the axial ratio of the outermost contour in Figure 2, after correction of that ratio for beam smearing, we obtain $\sin i \simeq 0.85$ (the axial ratios of the three lowest column density contours in Figure 2 are consistent with each other within an estimated accuracy of $20 \%$ ). The $V_{\text {rot }}$ corrected for inclination is then $10.6 \mathrm{~km} \mathrm{~s}^{-1}$. Thermal broadening and large-scale motions appear to contribute comparably to the linewidth of the integrated spectrum.

The preliminary VLA-C data discussed here are soon to be complemented by additional, approved observations in array configuration D (Cannon et al., in preparation) and by observations with the GMRT array (Chengalur et al., in preparation).

Table 1 summarizes the basic parameters of Leo $\mathrm{P}$ as follows:

- Coordinates of the HI emission centroid, for both Arecibo and the VLA maps; the source of all parameters labelled 'AO' in the table refer to the ALFALFA data set and those labelled 'VLA' refer to the VLA-C configuration data

- $V_{\odot}$ is the AO heliocentric velocity;

- $V_{g s r}=V_{l s r}+225 \sin l \cos b$ is the radial velocity in the Galactic Standard of Rest frame and $V_{l s r}$ is that in the Local Standard of Rest, with an assumed solar motion of $20 \mathrm{~km} \mathrm{~s}^{-1}$ towards $l=57^{\circ}$, $b=25^{\circ}$;

- $V_{L G}$ is the radial velocity in the LG dynamical rest frame, with respect to which the MW motion is of $316 \mathrm{~km} \mathrm{~s}^{-1}$ towards an apex of Galactic coordinates $(l, b)=,\left(93^{\circ},-4^{\circ}\right)$ (Karachentsev \& Makarov 1996);

- $W 50$ is the AO velocity width, measured at half power;

- $F_{H I}$ is the integrated flux density under the line of the AO feature;

- $R_{H I}$ is an (uncertain) estimate of the HI radius in $\mathrm{kpc}$, corresponding to $1^{\prime}$ in angular size along the major axis of the isophote at $5 \times 10^{19} \mathrm{HI}$ atoms $\mathrm{cm}^{-2}$ shown in Figure 2; the radius corresponding to the isophote at the level of half the peak HI column density is one-half the value of $R_{H I}$ in Table 1

- $M_{H I}$ is the HI mass, in solar units, as derived from $F_{H I}$;

- $V_{\text {rot }}$ is the maximum observed rotational velocity, corrected for disk inclination, as extracted from Figure 3;
Table 1

Properties of Leo P

\begin{tabular}{lc}
\hline \hline \multicolumn{1}{c}{ Parameter } & Value \\
\hline Right Ascension (J2000) AO & $10: 21: 45.0$ \\
Declination (J2000) AO & $18: 05: 01$ \\
Right Ascension (J2000) VLA & $10: 21: 44.8$ \\
Declination (J2000) VLA & $18: 05: 20$ \\
Galactic longitude & $219^{\circ} .654$ \\
Galactic latitude & $54^{\circ} .430$ \\
$V_{\odot}\left(\mathrm{km} \mathrm{s}^{-1}\right) \mathrm{AO}$ & $264 \pm 2$ \\
$V_{g s r}\left(\mathrm{~km} \mathrm{~s}^{-1}\right)$ & 177 \\
$V_{L G}\left(\mathrm{~km} \mathrm{~s}^{-1}\right)$ & 137 \\
$W_{50}\left(\mathrm{~km} \mathrm{~s}^{-1}\right) \mathrm{AO}$ & $24 \pm 2$ \\
$F_{H I}\left(\mathrm{Jy} \mathrm{km} \mathrm{s} \mathrm{k}^{-1}\right)$ & $1.31 \pm 0.04$ \\
$R_{H I}(\mathrm{kpc}) \mathrm{VLA}$ & $0.29 \pm 0.07 D_{M p c}$ \\
$M_{H I} / M_{\odot} \mathrm{AO}$ & $3.1 \times 10^{5} D_{M p c}^{2}$ \\
$V_{r o t}\left(\mathrm{~km} \mathrm{~s}^{-1}\right) \mathrm{VLA}$ & $10.6 \pm 2.2$ \\
$M_{d y n}\left(<R_{H I}\right) / M_{\odot}$ & $8 \times 10^{6} D_{M p c}$ \\
$M_{H I} / M_{*}$ & $2.6 \pm 0.5$ \\
& \\
\hline
\end{tabular}

- $M_{d y n}\left(<R_{H I}\right)$ is an estimate of the upper limit of the dynamical mass within the HI radius, on the assumption that the object is a self-gravitating system, computed via

$$
M_{d y n}\left(<R_{H I}\right) \simeq R_{H I} \sigma^{2} / G
$$

with $\sigma=W 50 / 2 \sqrt{2 \ln 2}$. The same result is obtained if $\sigma$ is replaced by $V_{\text {rot }}$;

- $M_{H I} / M_{*}$ is the ratio of the HI mass to the stellar mass; the latter was estimated by Rhode et al. (2013). The assumed error is not driven by uncertainties in the photometry, but rather by the assumed protocol to convert optical flux and color into mass.

\subsection{Preliminary Estimate of Distance}

The Tully-Fisher relation (Tully \& Fisher 1977) has been effective in the determination of cosmic distances. For galaxies with $V_{\text {rot }}>50 \mathrm{~km} \mathrm{~s}^{-1}$, that determination's accuracy is $15-20 \%$. In its usual format, as the scaling law between optical or near infrared luminosity vs. rotational velocity $V_{\text {rot }}$, it is not useful in practice with galaxies with small $V_{\text {rot }}$, both because the scatter grows with decreasing $V_{\text {rot }}$ below $50 \mathrm{~km} \mathrm{~s}^{-1}$, and the power law behavior characterizing faster rotators fades. However, in the form of a relation between observed baryonic mass and rotational width (hereafter referred to as the baryonic Tully-Fisher relation, or 'BTFR'), its power law behavior holds over more than five orders of magnitude in baryonic mass, with only moderate increase in scatter to the lowest $V_{\text {rot }}$ McGaugh (2012) has recently calibrated the BTFR to $V_{\text {rot }} \simeq 20 \mathrm{~km} \mathrm{~s}^{-1}$. A preliminary determination of the distance of Leo $\mathrm{P}$ can thus be obtained, with a modest extrapolation of the BTFR.

We define the observed baryonic mass in the same way as done by McGaugh (2012), i.e. adding the stellar mass with the gas mass as inferred from the HI observations, multiplied by 1.33 in order to account for Helium mass, assumed to be present in primordial abundance. We ignore the contribution of molecular gas. The stellar mass is derived from the optical data by Rhode et al.(2013). 


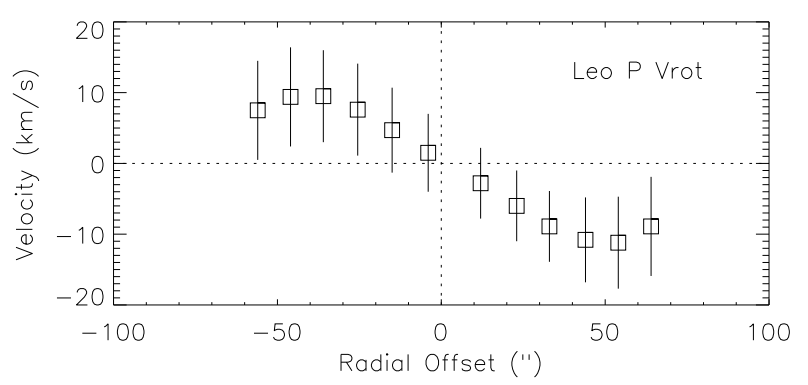

Figure 3. Rotation curve of Leo $\mathrm{P}$, extracted along the major axis of the preliminary VLA-C image shown in Fig. 2] uncorrected for disk inclination.

Thus the observed baryonic mass is

$$
\begin{aligned}
M_{b a r} & =1.33 M_{H I}+M_{*}=\left(1.33+2.6^{-1}\right) M_{H I} \\
& =\left(5.3 \times 10^{5} M_{\odot}\right) D_{M p c}^{2}
\end{aligned}
$$

McGaugh (2012) shows the BTFR in his Figure 1, which is partially reproduced in our Figure 4; the best fit power law to the data estimated by McGaugh (2012) is

$$
\log \left(M_{\text {bar }} / M_{\odot}\right)=2.01+3.82 \times \log V_{\text {rot }}
$$

with a mean scatter in $\log M_{b a r}$ of 0.24 dex. The scatter is however clearly increasing with decreasing $M_{b a r}$. For a value more appropriate to the low mass end of the BTFR we assume 0.36 dex, $50 \%$ higher than the mean value. The intersection of Leo P's $\log V_{\text {rot }}$ and the BTFR template relation yields a determination of the galaxy's distance: $1.3 \mathrm{Mpc}$. We coarsely estimate that the combination of errors in the estimate of the galaxy's disk inclination and rotational velocity is $20 \%$. Combining this with an assumed scatter about the BTFR of 0.36 dex results in an estimated error for the distance of $72 \%$, i.e. $D_{M p c}=1.3_{-0.5}^{+0.9}$. This is statistically in agreement with the optical estimates of Rhode et al. (2013) and McQuinn (private communication), discussed in section 3.1. We thus adopt a distance of $D_{M p c}=1.75$ for the remainder of this paper, for consistency in derived parameters with the papers by Rhode et al. (2013) and Skillman et al. (2013).

\section{DISCUSSION}

In a very useful recent compilation of galaxy properties in the LG and nearby groups, McConnachie (2012) separates LG members into 3 subgroups: MW satellites, M31 satellites and satellites of the LG as a whole. Galaxies that fall within the caustic curves of constant escape velocity in the $R-V$ plane of each subgroup - where $R$ is the distance of an object from the barycenter of the subgroup and V that object's velocity in the subgroup's rest frame - are assigned membership to the subgroup. At a distance of $1.75 \mathrm{Mpc}$, the location of Leo $\mathrm{P}$ in the $\mathrm{R}-\mathrm{V}$ planes of each subgroup is incompatible with membership in any of them. At that distance, its HI mass, as derived from the ALFALFA data, is $1.0 \times 10^{6} M_{\odot}$, its stelllar mass $3.6 \times 10^{5} M_{\odot}$, its $\mathrm{HI}$ radius $0.5 \mathrm{kpc}$ and its dynamical mass within that radius $1.4 \times 10^{7} M_{\odot}$.

It is interesting to note that the galaxy Leo I, which in terms of its sky location $\left(6^{\circ} .6\right.$ angular separation) and

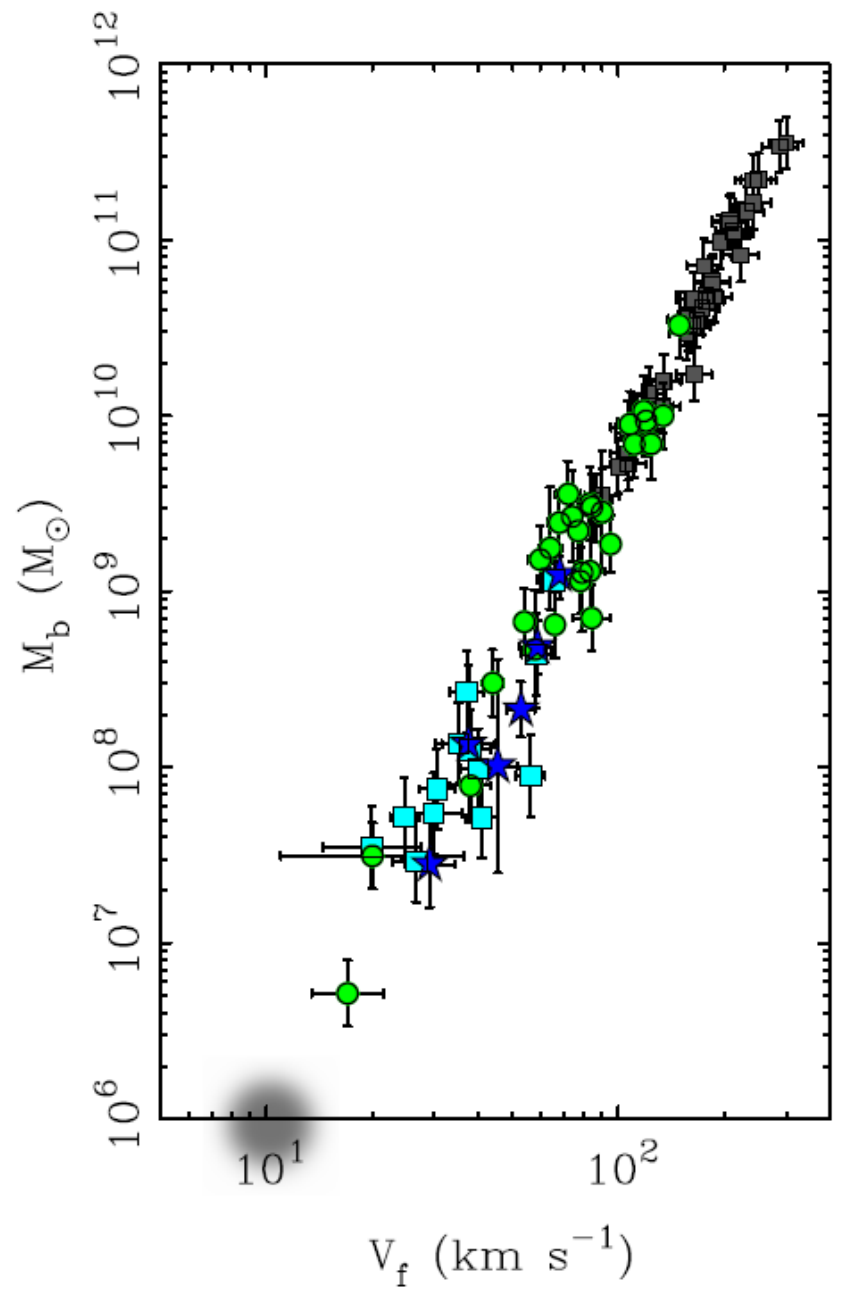

Figure 4. Location of Leo P (fuzzy symbol) on the BTFR, plotted on a reproduction of Fig. 1 of McGaugh (2012), showing a template of the baryonic BTFR of gas-rich galaxies (used with permission of the author and of the American Astronomical Society). The rotational velocity $V_{f}$ is measured in the same manner as our $V_{\text {rot }}$, from HI synthesis data.

heliocentric radial velocity $\left(282 \mathrm{~km} \mathrm{~s}^{-1} \mathrm{vs} .264\right.$ for Leo $\mathrm{P})$ would appear to be a close companion of Leo P, has a measured distance of $0.25 \mathrm{Mpc}$; that places Leo I within the $300 \mathrm{kpc}$ virial radius of the MW, assumed to have a mass of $10^{12} M_{\odot}$. Correcting its velocity for MW rotation, Leo I is traveling through the MW corona at $\geq 174$ $\mathrm{km} \mathrm{s}^{-1}$. The ALFALFA survey yields an upper limit to its HI mass of $6.0 \times 10^{3} M_{\odot}$. The nearest neighbors of Leo P appear to be the galaxies in the sparse NGC 3109 group, including Sextans B, located at $1.4 \mathrm{Mpc}$ from us and $\sim 350 \mathrm{kpc}$ from Leo P. The largest galaxy in that group, NGC 3109, has a V-band absolute magnitude of -14.9 and is located $44^{\circ}$ away from Leo P. The nearest giant galaxy to Leo $\mathrm{P}$ is the MW itself. Leo $\mathrm{P}$ is a bona fide gas bearing, star forming galaxy inhabiting a very lowmass halo, located in a low density environment lacking any massive companions, in the immediate vicinity of the LG. Its measured oxygen abundance is lower than that of any galaxy in the Local Volume and is similar to those of I Zw 18 and DDO 68. Leo P is however closer to us than the latter two by 11 and 7 times respectively. 
We use a distance to DDO 68 of $12.1 \pm 0.7 \mathrm{Mpc}$ based on recent TRGB data (A. Aloisi, personal communication). Unlike I Zw 18 and DDO 68, Leo P shows no evidence for tidal disturbances, although the asymmetry shown by the SE extension of the HI isophotes in Figure 2 could be the remnant of a past mild encounter. Forthcoming HI synthesis observations should help clarify this matter. There is however no evidence, either in optical or HI surveys, of any massive system in the vicinity of Leo P. In this object, the evolutionary history and extreme metallicity of a very low mass system can thus be studied in a laboratory environment "as clean as it gets".

The "dwarf galaxy problem", the observed underabundance of dwarf galaxies with respect to theoretical expectations, appears to be tightly related to their baryon deficiency (Papastergis et al. 2012). For low mass halos, the baryon deficiency is attributable to the shallow potential well of those systems, which makes them unable to retain their gas, which is lost due to either heating by the metagalactic UV field or to galactic winds, after episodic star formation events. This is shown effectively by the simulations of Hoeft et al. (2006), Hoeft \& Gottloeber (2010) and others. Heating of the IGM raises the Jeans mass, making IGM gas infall onto low mass systems ineffectual, yet these circumstances may change at later epochs, then gas accretion and star formation in a dwarf system may resume (Ricotti 2009). The transition from halos capable of retaining most of their baryons to those losing most of them takes place over a narrow range of halo masses. The mid-point for that transition is referred to as the 'characteristic halo mass' $M_{c}$. This would be the halo mass marking the onset of the dwarf galaxy problem. One should thus expect that the baryon-to-dark matter fraction of halos with mass $M_{h}<M_{c}$ would be measurable - if at all - at levels much lower than the cosmic fraction of $\sim 1 / 6$. Within this scenario, the thermal models of SMW02 are most useful in estimating the detectability of these objects. They indicate that halos with $M_{h}<10^{9} M_{\odot}$ can retain a small fraction of their baryons, albeit far below the cosmic baryon fraction as Nbody simulations suggest. Most of those baryons would be present in the form of a warm, ionized, thermal gas phase (WIM) with a temperature near $7000 \mathrm{~K}$ or higher, depending on the gas metallicity. An even smaller fraction of the baryon mass could be present within the inner region of the WIM, in the form of a warm neutral gas phase (WNM) or even in the form of a cold neutral phase (CNM), eventually capable of converting some of its gas into stars. According to those models, the neutral gas content of a $10^{9} M_{\odot}$ DM halo would thus not be around $10^{8} M_{\odot}$, but rather a few orders of magnitude lower, even below $10^{6} M_{\odot}$. Detection of such weak HI emitters would have been impossible by past or current wide field surveys beyond a few Mpc from us. Given their shallow potential wells, their gas content would be of marginal stability and their detection may require that they be located in environments sheltered from interaction with threatening neighbors and able to provide a measure of confining IGM pressure.

G+10 reported the discovery of a category of ultracompact HI HVCs which are plausible minihalo candidates, as shown by the SMW02 models. Leo P belongs to that category of objects. Leo P, Leo T - the LG transition galaxy which is located $417 \mathrm{kpc}$ from the MW, just outside the MW virial radius (Rvan-Weber et al. 2008) — and the dwarf companion of NGC 2903 (Irwin et al. 2009) may be the most extreme confirmed examples of gas-bearing minihalos. Others may exist with even fainter stellar counterparts, and some with no detectable starlight (or HI) at all. The catalog of UCHVCs as minihalo candidates of Adams et al. (2013), extracted from the ALFALFA data base, provides us with the targets that may help to shed further light on this category of dim galaxies.

The authors wish to thank the Director of the VLA for his positive response to a request for discretionary time (program code 12A-456; PI J. Cannon). We thank R. Koopmann, P. Troischt and their student members of the Undergraduate ALFALFA team for conducting the confirming L-band wide observations. The Undergraduate ALFALFA team is supported by NSF grants AST-0724918, AST-0725267, AST-0725380, AST0902211, and AST0903394. The ALFALFA work at Cornell is supported by NSF grants AST-0607007 and AST1107390 to RG and MPH and by grants from the Brinson Foundation. EAKA is supported by an NSF predoctoral fellowship. KLR is supported by NSF Faculty Early Career Development (CAREER) award AST-0847109 and JMC by NSF grant AST-1211683.

\section{REFERENCES}

Adams, E.A.K., Giovanelli, R. \& Haynes, M.P. 2013, ApJ, 768, 77 Blitz, L., Spergel, D.N., Teuben, P.J., Hartmann, P.J. \& Burton, W.B. 1999, ApJ, 514, 818

Braun, R. \& Burton, W.B. 1999, A\&A, 341, 437

Giovanelli, R., Haynes, M.P., Kent, B.R., et al. 2005, AJ, 130, 2598

Giovanelli, R., Haynes, M.P., Kent, B.R. \& Adams, E.A.K. 2010, ApJ, 708, L22 (G+10)

Haynes, M. P., Giovanelli, R., Martin, A.M., et al. 2011, AJ, 142, 170

Hoeft, M., Yepes, G., Gottloeber, S. \& Springel, V. 2006, MNRAS, 371, 401

Hoeft, M. \& Gottloeber, S. 2010, Advances in Astronomy, 2010, 87

Irwin, J., Hoffman, G. L., Spekkens, K., et al. 2009, ApJ, 692, 1447

Jorsater, S., \& van Moorsel, G. A. 1995, AJ, 110, 2037

Karachentsev, I.D. \& Makarov, D.A. 1996, AJ, 111, 794

Koposov, S., Belokurov, V., Evans, N.W. et al. 2008, ApJ, 686, 279

Klypin, A., Kravtsov, A.V., Valenzuela, O. \& Prada, F. 1999, ApJ, 522, 82

Martin, A.M., Papastergis, E., Giovanelli, R., et al. 2010, ApJ, 723,1359

McConnachie, A.W. 2012, AJ, 144, 4

McConnachie, A.W., Patton, D.R., Ellison, S.L. \& Simard, L. 2009, MNRAS, 395, 255

McGaugh, S.M. 2012, AJ, 143, 40

Papastergis, E., Martin, A. M., Giovanelli, R., \& Haynes, M. P. 2011, ApJ, 739, 38

Papastergis, E., Cattaneo, A., Huang, S. et al. 2012, 2012, ApJ, to appear and arXiv1208.5229

Peebles, P.J.E. 2001, ApJ 557, 495

Pisano, D.J., Barnes, D.G., Gibson, B.K., et al. 2007, ApJ, 662, 959

Rhode, K.L., Salzer, J.J., Haurberg, N., et al. 2013, AJ, 145, 149

Ricotti, M. 2009, MNRAS 392, L45

Ryan-Weber, E.V. et al. 2008, MNRAS, 384, 535

Saintonge, A. 2007, AJ, 133, 2087

Sand, D.J., Strader, J., Willman, B. et al. 2012, ApJ, 756, 795

Skillman, E.D., Salzer, J.J., Berg, D.A. et al. 2013, AJ (accepted) 
Sternberg, A., McKee, C.F. \& Wolfire, M.G. 2002, ApJS, 143, 419 Young, L.M. \& Lo, K.Y. 1996, ApJ, 462, 203 (SMW02)

Tully, R.B. \& Fisher, J.R. 1977, A\&A, 54, 661

Warren, S.R., Skillman, E.D., Stilp, A.M. et al. 2012, ApJ, 757, 84 